\title{
openheart Age-specific atrial fibrillation incidence, attributable risk factors and risk of stroke and mortality: results from the MORGAM Consortium
}

\author{
Bente Morseth (D) ," Bastiaan Geelhoed, ${ }^{2}$ Allan Linneberg, ${ }^{3,4}$ Lars Johansson, ${ }^{5}$ \\ Kari Kuulasmaa (1) , ${ }^{6}$ Veikko Salomaa (1) , ${ }^{6}$ Licia lacoviello (1) , 7,8 \\ Simona Costanzo (D) , ${ }^{8}$ Stefan Söderberg (D) , ${ }^{5}$ Teemu J Niiranen, ${ }^{6,9}$ \\ Julie K K Vishram-Nielsen, ${ }^{3,10}$ Inger Njølstad, ${ }^{11}$ Tom Wilsgaard, ${ }^{11}$ \\ Ellisiv B Mathiesen, ${ }^{12,13}$ Maja-Lisa Løchen (1) ,11 Tanja Zeller, ${ }^{2,14}$ \\ Stefan Blankenberg, ${ }^{2,14}$ Francisco M Ojeda, ${ }^{2}$ Renate B Schnabel (D) , 2,14 On behalf \\ of the MORGAM consortium
}

\begin{abstract}
- Additional supplemental material is published online only. To view, please visit the journal online (http://dx.doi.org/10.
\end{abstract} 1136/openhrt-2021-001624).

To cite: Morseth B, Geelhoed B, Linneberg A, et al. Age-specific atrial fibrillation incidence, attributable risk factors and risk of stroke and mortality: results from the MORGAM Consortium. Open Heart 2021;8:e001624. doi:10.1136/ openhrt-2021-001624

Received 13 February 2021 Accepted 13 July 2021

Check for updates

(C) Author(s) (or their employer(s)) 2021. Re-use permitted under CC BY-NC. No commercial re-use. See rights and permissions. Published by BMJ.

For numbered affiliations see end of article.

Correspondence to Dr Bente Morseth; bente. morseth@uit.no

\section{ABSTRACT}

Background The main aim was to examine age-specific risk factor associations with incident atrial fibrillation (AF) and their attributable fraction in a large European cohort. Additionally, we aimed to examine risk of stroke and mortality in relation to new-onset AF across age. Methods We used individual-level data $(\mathrm{n}=66951$, $49.1 \%$ men, age range $40-98$ years at baseline) from five European cohorts of the MOnica Risk, Genetics, Archiving and Monograph Consortium. The participants were followed for incident AF for up to 10 years and the association with modifiable risk factors from the baseline examinations (body mass index (BMI), hypertension, diabetes, daily smoking, alcohol consumption and history of stroke and myocardial infarction (MI)) was examined. Additionally, the participants were followed up for incident stroke and all-cause mortality after new-onset AF. Results AF incidence increased from 0.9 per 1000 person-years at baseline age 40-49 years, to 17.7 at baseline age $\geq 70$ years. Multivariable-adjusted Cox models showed that higher BMI, hypertension, high alcohol consumption and a history of stroke or MI were associated with increased risk of AF across age groups $(p<0.05)$. Between $30 \%$ and $40 \%$ of the AF risk could be attributed to BMI, hypertension and a history of stroke or MI. New-onset AF was associated with a twofold increase in risk of stroke and death at ages $\geq 70$ years $(p \leq 0.001)$. Conclusion In this large European cohort aged 40 years and above, risk of AF was largely attributed to BMI, high alcohol consumption and a history $\mathrm{Ml}$ or stroke from middle age. Thus, preventive measures for AF should target risk factors such as obesity and hypertension from early age and continue throughout life.

\section{INTRODUCTION}

Atrial fibrillation (AF) has become a disease with significant public health impact, estimated to affect 20 million men and 12 million

\section{Key questions}

What is already known about this subject?

- Modifiable risk factors for atrial fibrillation (AF), such as obesity, hypertension, diabetes, smoking and previous cardiovascular disease, may together account for more than $50 \%$ of the AF burden. However although risk factors for AF have been extensively examined, limited attention has been devoted to their attributable fraction in relation to age.

What does this study add?

- In this large cohort of adult and elderly European men and women, risk of AF was primarily attributed to body mass index, hypertension and previous stroke or myocardial infarction, together explaining $30 \%-40 \%$ of the AF risk. Population attributable risk varied with age, with a substantial AF burden attributed to body mass index at ages 40-69 years, whereas population attributable risk for hypertension was highest and significant only at ages $\geq 60$ years. The AF risk attributed to previous myocardial infarction or stroke increased slightly with age.

How might this impact on clinical practice?

- Increased understanding of the relative contribution of modifiable risk factors to the development of $\mathrm{AF}$, as well as complications of $\mathrm{AF}$ across age groups, would help target prevention and allocate healthcare resources specific to age group needs.

women worldwide. ${ }^{1}$ Major complications of $\mathrm{AF}$ include stroke, ${ }^{2}$ hospitalisations ${ }^{3}$ and mortality. ${ }^{4}$ Several predictors for $\mathrm{AF}$ have been described, including modifiable risk factors such as obesity, hypertension, diabetes, smoking and previous cardiovascular disease ${ }^{5-7}$ which may together account for more than $50 \%$ of the AF burden. 
$\mathrm{AF}$ incidence increases with age $\mathrm{e}^{9-11}$ and is commonly related to the presence of cardiovascular risk factors or disease in older individuals. ${ }^{12}$ On the other hand, transition to unhealthy metabolic risk factor constellations occurs earlier in life, ${ }^{13}$ and a recent study suggests that even in early-onset AF, $90 \%$ of individuals had metabolic risk factors and comorbidities. ${ }^{14}$ Although several large cohort studies have addressed the role of lifestyle risk factor for $\mathrm{AF}^{1516}$ and potential sex differences, ${ }^{17}$ there is a lack of research on the impact of the risk factors in relation to age at $\mathrm{AF}$ onset.

Increased understanding of the contribution of modifiable risk factors in relation to age at AF onset, as well as complications of AF across age groups, would help target prevention and allocate healthcare resources specific to age group needs. The MOnica Risk, Genetics, Archiving and Monograph (MORGAM) consortium provides a unique opportunity to expand the understanding of $\mathrm{AF}$ risk factors and complications from middle aged to older individuals. The main aim of this study was to examine age-specific risk factor associations with incident $\mathrm{AF}$ and their attributable fraction in European adults and elderly. Furthermore, we aimed to examine the risk of stroke and all-cause mortality after new-onset AF at different ages.

\section{METHODS}

\section{Study population}

This study is based on the MORGAM consortium, which is a multinational collaborative initiative addressing cardiovascular biomarkers, risk factors and endpoints using pooled and harmonised data from European communitybased cohorts. ${ }^{18}$ In this study, individual-level data from five cohort studies in the MORGAM consortium ${ }^{18}$; DANMONICA (Denmark), ${ }^{19}$ FINRISK (Finland) ${ }^{20}$ Moli-sani (Italy), ${ }^{21}$ Northern Sweden MONICA, ${ }^{22}$ and the Troms $\varnothing$ Study (Norway) ${ }^{23}$ were included. Cohort-specific procedures for enrolment and follow-up are provided in the online supplemental D1. In brief, all included cohort studies are population-based with repeated surveys. The studies included in MORGAM are population-based studies covering a specific region in several European countries, recruiting from birth cohorts or random sampling from a national or local population register. Most studies do not specify exclusion criteria.

In total, 66951 individuals from the five MORGAM cohort studies were included in our analyses, after exclusion due to left censoring $(\mathrm{n}=448)$, prevalent $\mathrm{AF}$ at baseline $(n=879)$ and/or missing baseline AF information $(\mathrm{n}=6757)$. Only individuals aged $\geq 40$ years at baseline were included in order to harmonise the age range.

\section{Risk factors and biomarkers}

Data on risk factors were available from the baseline examinations, which were conducted between 1982 and 2010. Systolic blood pressure (SBP), total cholesterol, weight and height were measured by standardised methods and body mass index (BMI) $\left(\mathrm{kg} / \mathrm{m}^{2}\right)$ was calculated. Information on daily smoking, average alcohol consumption, diabetes, antihypertensive medication, history of stroke and myocardial infarction (MI) was self-reported. Harmonised data from the various studies were transferred to the MORGAM Data Centre in Helsinki. ${ }^{24}$

Biomarkers were available for $39 \%-49 \%$ of the participants (available in FINRISK, Moli-sani, Northern Sweden, and Dan-MONICA) (online supplemental tables S1S5). Measurements from stored blood samples include C reactive protein (CRP) ( $n=32893)$, which was determined by latex immunoassay CRP16 (Abbott, Architect c8000), and N-terminal-pro B-type natriuretic peptide (Nt-pro-BNP) (n=26 048, measured on the ELECSYS 2010 platform using an electrochemiluminescence immunoassay (Roche Diagnostics). Analyses of estimated glomerular filtration rate (eGFR), CRP, and Nt-proBNP are described in detail elsewhere. ${ }^{25}$

\section{Follow-up for AF, stroke and mortality}

Incident AF, non-fatal and fatal stroke events, and death during follow-up were identified by linkage to regional or national hospital discharge registries, diagnosis registries, and causes of death registries, which were also screened for incident $\mathrm{AF}$ as a comorbidity of individuals who died of other causes. Follow-up lasted from the date of the baseline examinations to the end of 2010 (DANMONICA, Troms $\varnothing$ and FINRISK) or 2011 (Moli-sani and Northern Sweden), truncated at 10 years for the data analysis (median (IQR): 10.0 (4.8-10.0) years).

\section{Statistical analyses}

For each age group (40-49, 50-59, 60-69 and $\geq 70$ years at baseline), AF incidence rates were calculated as the number of AF cases per 1000 person-years within the 10 -year follow-up period. The association between risk factors and incident $\mathrm{AF}$ within each age stratum was estimated as HR with 95\% CI using Cox regression. The goodness-of-fit of all Cox models was checked with respect to the proportional hazard $(\mathrm{PH})$ assumption. In order to assure that the $\mathrm{PH}$ assumption was valid during the Cox regressions, interactions with time were adjusted for when needed. The $\mathrm{PH}$ assumptions were tested using the Schoenfeld residuals. Prevalent AF cases were excluded from the analysis. Multivariable-adjusted models include adjustment for age, sex, BMI, hypertension ( $\mathrm{SBP} \geq 140 \mathrm{~mm} \mathrm{Hg}$ and/or taking antihypertensive drugs), total serum cholesterol, smoking, history of diabetes, alcohol consumption and study site. We set the follow-up duration to a fixed maximum value (10 years) to avoid large differences in follow-up. Missing data were handled by using available cases.

Population attributable fraction (PAF) was calculated for each categorical risk factor. In each age group, the PAF for a risk factor was estimated using the fully adjusted estimated HRs and the following equation ${ }^{26}$ :

$$
\mathrm{PAF}_{\mathrm{i}}=\mathrm{pd}_{\mathrm{i}} *\left(\mathrm{HR}_{\mathrm{i}}-1\right) / \mathrm{HR}_{\mathrm{i}}
$$


where $\mathrm{PAF}_{\mathrm{i}}$ is the PAF of the $\mathrm{i}$-th category, $\mathrm{HR}_{\mathrm{i}}$ is the fully adjusted $\mathrm{HR}$ for the $\mathrm{i}$-th category, $\mathrm{pd}_{\mathrm{i}}$ is the proportion of those in the i-th category among the cases during the first 5 years of the follow-up. The total PAF of a risk factor was calculated as the sum of all $\mathrm{PAF}_{i}$ over $\mathrm{i}=1$ to $n$, where $\mathrm{n}$ is the number of categories of the risk factor in question. For each $\mathrm{PAF}_{\mathrm{i}}$ and PAF, a 95\% CI was calculated using bootstrapping. Bootstrapping was also used to estimate the $p$ values for the differences between PAFs of different age groups for the same risk factor. To decide whether a difference was significant, a (Bonferroni-corrected) alpha level of $0.05 / \mathrm{N}$ was used, where $\mathrm{N}$ is the number of comparisons per risk factor category level (there are four age groups, so $\mathrm{N}$ is $(4 \times 4-4) / 2=6$, so alpha $=0.05 / 6=0.008)$.

In online supplemental table $\mathrm{S} 6$, second-order interactions between covariates were included if they reached significance (taking into account multiple testing). Interactions with time since baseline (included in the analysis as a time-varying covariate) were included when needed to avoid violations of the $\mathrm{PH}$ assumption. The PAF analyses used fully adjusted HRs.

In order to estimate the HRs of new-onset AF for stroke and mortality by age-decades, time-dependent multiple adjusted Cox regressions were performed in each age stratum where incident $\mathrm{AF}$ was used to predict time to event (where event is (1) stroke and (2) mortality). For the stroke analysis, cases with previous stroke history were excluded.

Analyses were performed in R V.3.5.3, in which Cox regressions were performed using the survival package. We assumed a two-tailed $\mathrm{p}<0.05$ as statistically significant.

\section{RESULTS}

The median age at baseline was 53.5 years (IQR 46.461.2), ranging from 40 to 98 years, and $49.1 \%$ of the 66 951 participants were men (table 1). The prevalence of previous disease (diabetes and history of MI or stroke) was low $(<6 \%)$, although more than half of the participants $(52.2 \%)$ had an SBP $\geq 140 \mathrm{~mm} \mathrm{Hg}$ or were taking antihypertensive drugs. About one-fourth (26.0\%) of the participants smoked daily. Study characteristics by cohort are shown in online supplemental tables S1-S5. Risk factor distributions at baseline were in general more favourable at younger age (table 2). Prevalence of hypertension, overweight, diabetes and previous MI or stroke increased with age, whereas smoking decreased gradually by age, and daily consumption of alcohol showed a stable pattern across all age groups.

Over a follow-up time of up to 10.0 years, 2021 (3.0\%) cases of AF were detected. AF incidence rate increased gradually from 0.9 per 1000 person-years at age $40-49$ years to 17.7 at the age of $\geq 70$ years. Adjusted HR for $\mathrm{AF}$ increased exponentially with age and was 13.5 times higher (HR 13.53, 95\% CI 10.98 to 16.68 ) at ages $\geq 70$ compared with 40-49 years (figure 1).

Age-stratified unadjusted risk of AF for each risk factor is shown in online supplemental table S7. Age-stratified
Table 1 Baseline characteristics of the pooled MORGAM cohort $(n=66951)$

\begin{tabular}{llr}
\hline Characteristics & $\begin{array}{l}\text { Median (IQR) or } \\
\mathbf{n}(\%)\end{array}$ & Missing values \\
\hline Age (years) & $53.5(46.4-61.2)$ & $0(0.0 \%)$ \\
\hline Sex (n (\%) men) & $32855(49.1)$ & $0(0.0 \%)$ \\
\hline BMI (kg/m²) & $26.4(23.9$ to 29.5$)$ & $480(0.7 \%)$ \\
\hline Systolic blood pressure (mm Hg) & $137(124$ to 152$)$ & $459(0.7 \%)$ \\
\hline Total serum cholesterol (mmol/L) & $5.9(5.2$ to 6.8$)$ & $669(1.0 \%)$ \\
\hline Daily smoker & $17305(26.0)$ & $370(0.6 \%)$ \\
\hline History of diabetes & $3526(5.3)$ & $83(0.1 \%)$ \\
\hline Taking antihypertensive drugs & $11977(19.2)$ & $4576(6.8 \%)$ \\
\hline Hypertension† & $33470(52.2)$ & $2781(4.2 \%)$ \\
\hline Average daily alcohol consumption (g) & $3.0(0.0$ to 12.0$)$ & $11777(17.6 \%)$ \\
\hline History of Ml or stroke & $3625(5.4)$ & $62(0.1 \%)$ \\
\hline C reactive protein (mg/L) & $1.4(0.7$ to 2.9$)$ & $34058(50.9 \%) \ddagger$ \\
\hline Nt-proBNP (pg/mL) & $52(27$ to 96$)$ & $40903(61.1 \%) \S$ \\
\hline log10 (Nt-proBNP (pg/mL)) & $1.7(1.4$ to 2.0$)$ & $40903(61.1 \%) \S$ \\
\hline Creatinine (mg/dL) & $0.8(0.7$ to 0.9$)$ & $34065(50.9 \%)$ 丹 \\
\hline eGFRף & $94.8(84.0$ to 103.5$)$ & $34065(50.9 \%) \ddagger$ \\
\hline
\end{tabular}

*Mean of two measurements.

†Systolic blood pressure $\geq 140 \mathrm{~mm} \mathrm{Hg}$ and/or taking antihypertensive drugs. ¥Missing by design in the Troms $\varnothing$ Study and partly in other studies than the 1997 cohort of FINRISK (online supplemental tables S2 and S4).

§Missing by design in DAN-MONICA and the Tromsø Study, and partly in other studies except the 1997 cohort of FINRISK (online supplemental tables S1, S2 and S4).

IUsing the Chronic Kidney Disease Epidemiology Collaboration formula with creatinine.

BMI, body mass index; eGFR, estimated glomerular filtration rate; MI, myocardial infarction; MORGAM, MOnica Risk, Genetics, Archiving and Monograph; Nt-proBN, N-terminal-pro B-type natriuretic peptide.

multivariable-adjusted Cox regression models showed that BMI and a history of MI or stroke were consistently associated with increased risk of $\mathrm{AF}$ in all age groups $(\mathrm{p}<0.001)$ (table 3$)$, whereas high alcohol consumption, total serum cholesterol, and hypertension increased the risk of AF only at certain ages. For each $5 \mathrm{~kg} / \mathrm{m}^{2}$ increase in BMI, risk of AF increased significantly with $37 \%-41 \%$ $(p \leq 0.001)$ at ages $40-69$ years. At the age of $\geq 70$ years, AF risk was still positively associated with BMI (HR 1.17, $95 \%$ CI 1.06 to 1.29 ), but the risk was lower than at younger ages (difference from ages 40-69: $\mathrm{p} \leq 0.033$ ). A history of MI or stroke significantly increased the risk of AF ( $\mathrm{p} \leq 0.001$ at all ages), with highest HRs at age 40-49 years (HR 4.60, 95\% CI 2.56 to 8.27; $\mathrm{p} \geq 0.011$ compared with other age groups). High alcohol consumption significantly increased the risk of AF by $13 \%-18 \%$ at ages up to 70 years $(\mathrm{p}<0.05)$, but not in participants $\geq 70$ years. Total serum cholesterol decreased the risk of $\mathrm{AF}$ by $4 \%-11 \%$, but significantly only at ages $50-69$ years $(p<0.05)$, whereas hypertension increased the risk of AF by $19 \%-40 \%$, although significantly only at older ages ( $\geq 60$ years) $(p<0.05)$. Smoking, CRP and eGFR were not significantly associated with $\mathrm{AF}$, whereas participants with Nt-pro-BNP levels $\geq 900 \mathrm{pg} / \mathrm{mL}$ showed higher AF risk at all ages $(\mathrm{p} \leq 0.001)$. 
Table 2 Risk factor distribution by age groups at baseline

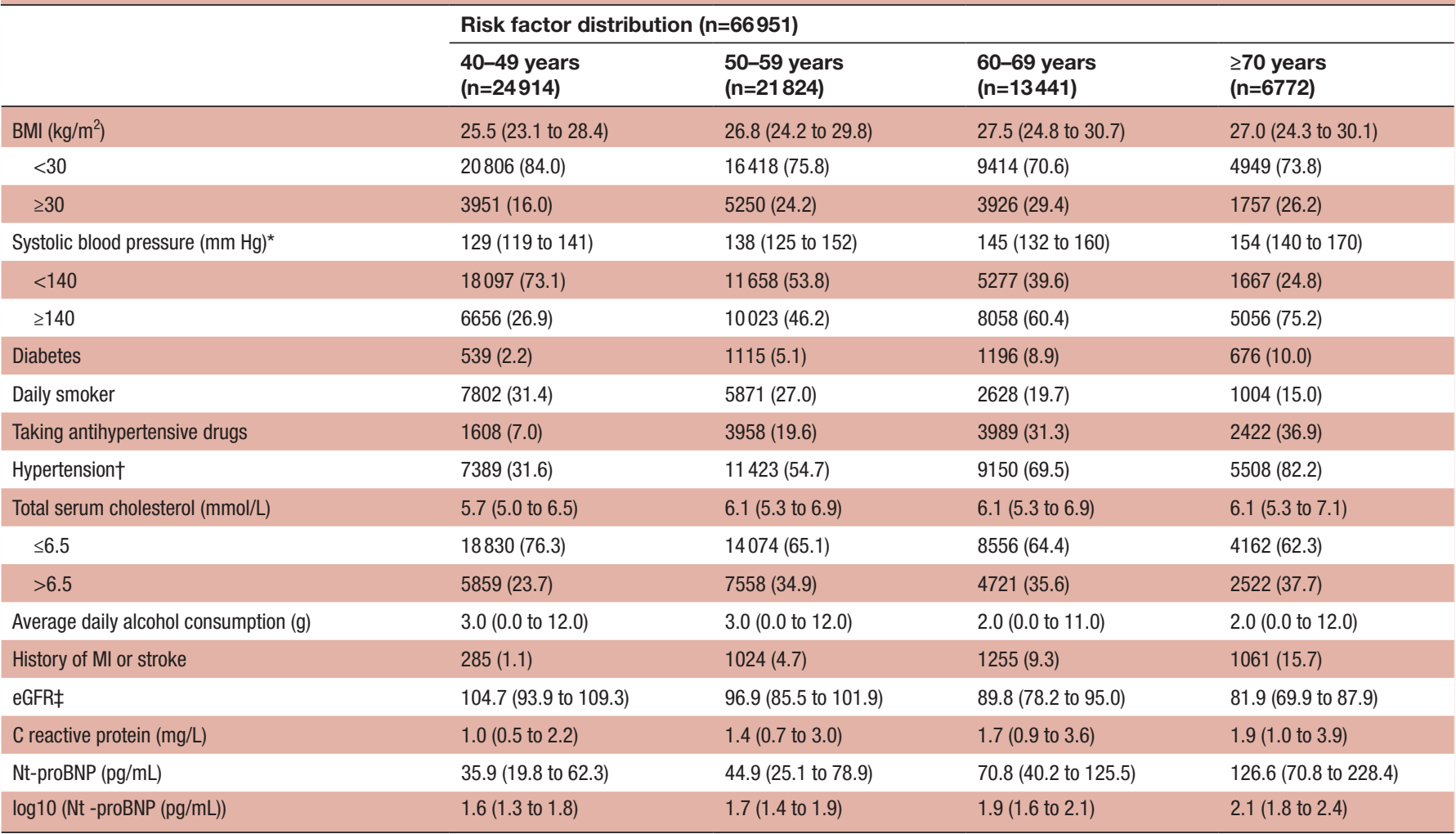

Continuous variables are presented as median and IQR (25th and 75th percentile), binary variables as absolute and relative frequencies.

*Mean of two measurements.

†Systolic blood pressure $\geq 140 \mathrm{~mm} \mathrm{Hg}$ and/or taking antihypertensive drugs.

fUsing the Chronic Kidney Disease Epidemiology Collaboration formula with creatinine.

$\mathrm{BMI}$, body mass index; eGFR, estimated glomerular filtration rate; MI, myocardial infarction; Nt-pro-BN, N-terminal-pro B-type natriuretic peptide.

Table 4 shows risk of $\mathrm{AF}$ attributed to each modifiable risk factor in age strata (ie, PAF for 5-year incident AF). Among lifestyle risk factors, BMI contributed to $8 \%-18 \%$ of the $\mathrm{AF}$ risk at ages $<70$, but at older ages, the relative attribution to BMI was low and non-significant $(4.0 \%$, $95 \%$ CI -1.31 to 9.20$)$. Hypertension showed significant PAFs of $20 \%$ only at ages $\geq 60$ years, whereas diabetes

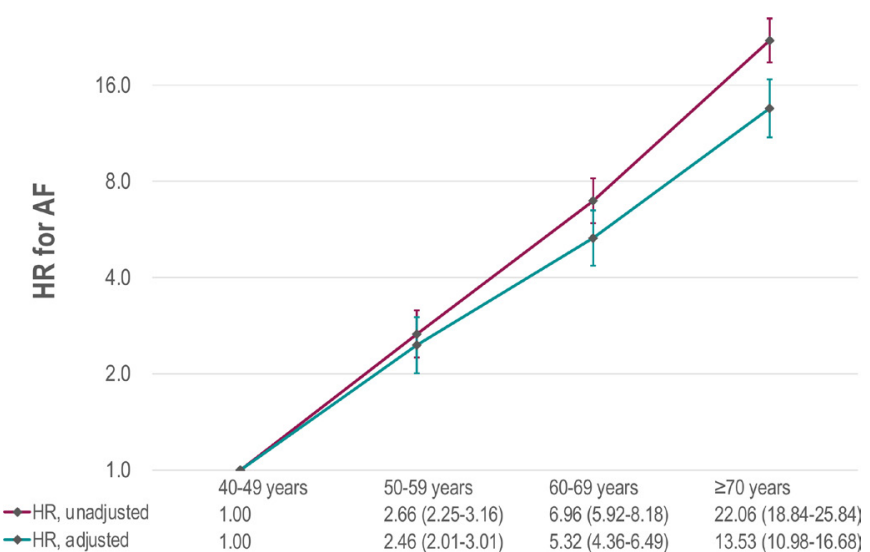

Figure 1 Risk of $\mathrm{AF}$ by age groups, presented as $\mathrm{HR}$ with $95 \% \mathrm{Cl}$. HRs were presented on a log scale with base 2, adjusted for sex, BMI, SBP, total serum cholesterol, daily smoking, history of diabetes, antihypertensive drugs, daily alcohol consumption, and study site. AF, atrial fibrillation; $\mathrm{BMI}$, body mass index; SBP, systolic blood pressure. showed a significant PAF only at ages $\geq 70$ years $(6.0 \%$, $95 \%$ CI 2.21 to 9.68 ). A history of stroke or MI contributed significantly to AF risk in all age groups with PAFs increasing gradually from $5 \%$ to $10 \%$. Smoking and alcohol intake showed modest and non-significant PAFs. In general, $30 \%-40 \%$ of the AF risk could be attributed to BMI, hypertension and a history of stroke or MI.

During the follow-up, $8.4 \%(\mathrm{n}=5622)$ participants died, whereas $3.1 \%(n=2055)$ were diagnosed with stroke. Newonset $\mathrm{AF}$ was associated with a 1.8-fold to 2.6-fold increase in risk of death at ages $\geq 60$ years (multivariable-adjusted $\mathrm{p} \leq 0.001$, figure 2 ) and with a twofold risk of stroke at ages $\geq 70$ years (HR 2.02, 95\% CI 1.46 to 2.79 , figure 2 ).

\section{DISCUSSION}

In this large, community-based cohort, risk of new-onset $\mathrm{AF}$ was associated with several lifestyle-related risk factors such as overweight, hypertension, high alcohol consumption and a history of MI or stroke. In all age groups, AF burden was largely attributable to BMI, hypertension, and previous stroke or MI, together explaining 30\%-40\% of the AF risk. However, their PAF varied with age, as we found a substantial AF burden attributed to BMI at ages 40-69 years, but low PAF for BMI among the oldest. PAF for hypertension was highest and significant only at ages $\geq 60$ years, whereas PAF for previous MI or stroke showed 
Table 3 Multivariable-adjusted HR for AF by age groups

\begin{tabular}{|c|c|c|c|c|}
\hline & \multicolumn{4}{|c|}{ Multivariable-adjusted HR for AF } \\
\hline & $\begin{array}{l}40-49 \text { years } \\
(n=24914)\end{array}$ & $\begin{array}{l}50-59 \text { years } \\
(n=21824)\end{array}$ & $\begin{array}{l}60-69 \text { years } \\
(n=13441)\end{array}$ & $\begin{array}{l}\geq 70 \text { years } \\
(n=6772)\end{array}$ \\
\hline BMI (per $5 \mathrm{~kg} / \mathrm{m}^{2}$ increase) & $1.41(1.18$ to $1.68,<0.001)$ & $1.37(1.23$ to $1.53,<0.001)$ & $1.37(1.25$ to $1.50,<0.001)$ & $1.17(1.06 \text { to } 1.29,0.001)^{*}$ \\
\hline Diabetes & $0.57(0.18$ to $1.80,0.337)$ & $1.48(1.04$ to $2.09,0.029)$ & $1.27(0.96$ to $1.66,0.090)$ & $1.59(1.23$ to $2.06,<0.001)$ \\
\hline Daily smoker & $1.13(0.79$ to $1.63,0.501)$ & $1.14(0.89$ to $1.45,0.305)$ & $1.16(0.92$ to $1.46,0.220)$ & $1.00(0.78$ to $1.27,0.988)$ \\
\hline Hypertension† & $1.40(0.98$ to $2.00,0.068)$ & 1.19 (0.94 to $1.51,0.138)$ & $1.32(1.07$ to $1.64,0.011)$ & $1.29(1.02$ to $1.63,0.035)$ \\
\hline $\begin{array}{l}\text { Total serum cholesterol (per mmol/L } \\
\text { increase) }\end{array}$ & 0.91 (0.78 to $1.06,0.242)$ & 0.91 (0.83 to $0.99,0.039)$ & 0.89 (0.83 to $0.97,0.004)$ & 0.96 (0.89 to $1.03,0.283)$ \\
\hline $\begin{array}{l}\text { Average daily alcohol consumption } \\
\text { (per } 20 \mathrm{~g} \text { increase) }\end{array}$ & $1.18(1.02$ to $1.36,0.025)$ & $1.17(1.05$ to $1.30,0.005)$ & $1.13(1.00$ to $1.28,0.049)$ & $0.92(0.79$ to $1.08,0.327) \ddagger$ \\
\hline History of Ml or stroke & $4.60(2.56$ to $8.27,<0.001) \S$ & $1.93(1.40$ to $2.67,<0.001)$ & $1.68(1.32$ to $2.13,<0.001)$ & $1.57(1.27$ to $1.94,<0.001)$ \\
\hline eGFRף & $1.07(0.90$ to $1.26,0.430)$ & $0.93(0.84$ to $1.03,0.193)$ & $1.00(0.92$ to $1.09,0.984)$ & $0.88(0.81$ to $0.96,0.005)$ \\
\hline $\mathrm{C}$ reactive protein (mg/L) & 0.94 (0.83 to $1.06,0.295)$ & $1.00(0.97$ to $1.03,0.960)$ & 1.01 (0.99 to $1.02,0.245)$ & 1.01 (0.99 to $1.02,0.348)$ \\
\hline Nt-pro-BNP (per 10-fold increase) & $5.51(2.70$ to $11.24,<0.001)$ & 4.80 (3.29 to $6.98,<0.001)$ & 5.46 (4.08 to $7.31,<0.001)$ & $7.58(5.62-10.24,<0.001)$ \\
\hline
\end{tabular}

Values are $\mathrm{HR}(95 \% \mathrm{Cl}, \mathrm{p}$ value).

Adjusted for age, sex, BMI, total serum cholesterol, daily smoking, diabetes, hypertension, daily alcohol consumption and study site.

${ }^{*} \mathrm{P} \leq 0.033$ for difference between ages $\geq 70$, and $50-59$ and $60-69$, respectively.

†Systolic blood pressure $\geq 140 \mathrm{~mm} \mathrm{Hg}$ and/or taking antihypertensive drugs.

$\neq \mathrm{p} \leq 0.047$ for difference between ages $\geq 70$ and the other age groups.

$\S \mathrm{P} \leq 0.011$ for difference between ages $40-49$ and the other age groups.

IUsing the Chronic Kidney Disease Epidemiology Collaboration formula with creatinine.

AF, atrial fibrillation; BMI, body mass index; eGFR, estimated glomerular filtration rate; Ml, myocardial infarction; Nt-proBN, N-terminal-pro B-type natriuretic peptide.

a slight increase with age. Finally, we found that newly diagnosed AF was associated with a twofold increase in risk of death and stroke but only at older ages.

\section{AF incidence, risk factors and attributable risks}

We observed a substantial increase in AF incidence with age that is comparable to age-specific incidence rates in the Rotterdam Study ${ }^{11}$ and the Reykjavik study ${ }^{9}$ but somewhat lower than the Framingham Study incidence rates. ${ }^{10}$ The increasing incidence of $\mathrm{AF}^{9-11}$ highlights the importance of modifiable risk factors, ${ }^{27} 28$ which can be targeted and may contribute to prevention or deferral of AF onset.
In the present study, several modifiable risk factors such as BMI, previous MI or stroke, hypertension and alcohol consumption were associated with increased risk of $\mathrm{AF}$, the first two consistently in all age groups. These observations are largely in agreement with a study of 10 million individuals in a national Korean health insurance database ${ }^{29}$ which showed that modifiable risk factors such as BMI, high alcohol intake, and hypertension increased the risk of AF in all age groups.

Our study extends previous research by addressing the proportion of incident $\mathrm{AF}$ that could be attributed to a

Table 4 Multivariable-adjusted population attributable fraction for AF by age groups

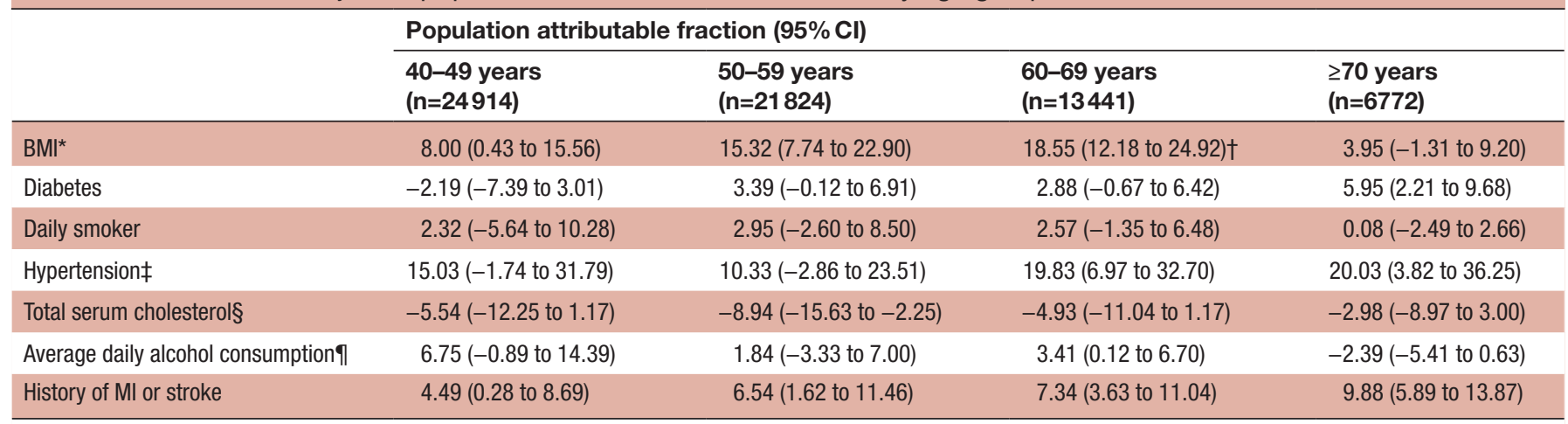

Adjusted for age, sex, BMI, total serum cholesterol, daily smoking, diabetes, hypertension, daily alcohol consumption and study site.

${ }^{*}$ Reference category $\leq 30 \mathrm{~kg} / \mathrm{m}^{2}$.

†Significantly different from age $\geq 70, p=0.002$; all other between-group $p>0.008$.

$\ddagger$ Systolic blood pressure $\geq 140 \mathrm{~mm} \mathrm{Hg}$ and/or taking antihypertensive drugs.

§Reference category $\leq 6.5 \mathrm{mmol} / \mathrm{L}$.

qReference category $<20 \mathrm{~g}$ for women, $<40 \mathrm{~g}$ for men.

$\mathrm{AF}$, atrial fibrillation; $\mathrm{BMI}$, body mass index; $\mathrm{MI}$, myocardial infarction. 


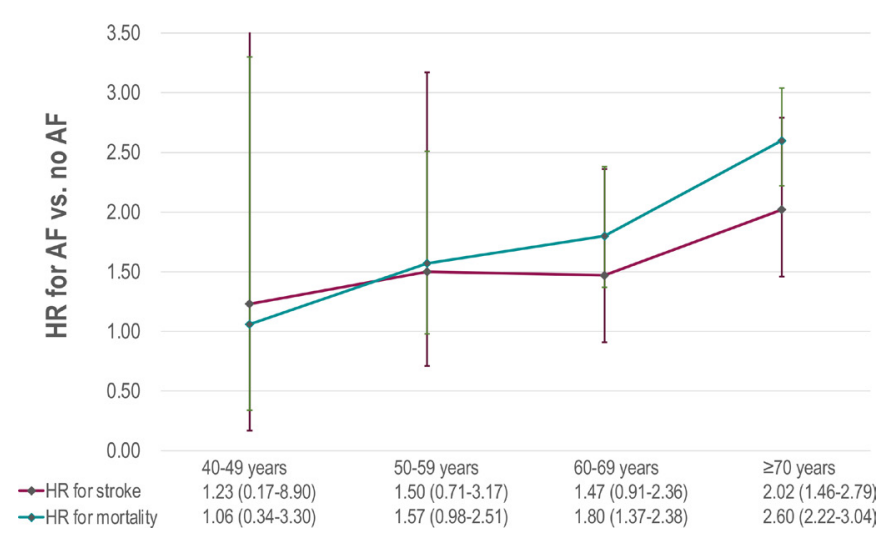

Figure 2 Risk of stroke and mortality according to new-onset AF versus no AF, presented as HR with $95 \% \mathrm{Cl}$. HRs were adjusted for age, sex, BMI, SBP, total serum cholesterol, daily smoking, history of diabetes, antihypertensive drugs, daily alcohol consumption, and study site. AF, atrial fibrillation; BMI, body mass index; SBP, systolic blood pressure.

risk factor at different ages. The proportion of AF risk attributed to BMI increased with age up to 70 years, with PAFs of $15 \%-18 \%$ which is comparable to the Atherosclerosis Risk in Communities Study. ${ }^{8}$ At ages $\geq 70$ years, PAF for BMI was low and not statistically significant, suggesting that at older ages new-onset $\mathrm{AF}$ is primarily attributed to factors other than BMI. The risk of AF attributed to hypertension was high at all ages with the highest attributable fractions in participants $\geq 60$ years, accounting for $20 \%$ of incident $\mathrm{AF}$, which is similar to results reported from other studies. ${ }^{830}$ As expected at population level, the risk of $\mathrm{AF}$ attributed to previous MI or stroke increased with age, possibly due to the observed age-related increase in prevalence of MI and stroke. Our findings underline the need to prevent and monitor cardiovascular risk factors and disease onset at an early age in order to prevent or postpone $\mathrm{AF}$ and its sequelae.

\section{Stroke and mortality after AF onset}

Increased risks of stroke and mortality are well-known complications of $\mathrm{AF}^{17}$ However, age-specific complications of AF are scarcely examined in large cohorts, although clinically, older age is a strong predictor of stroke in AF patients, ${ }^{31}$ manifested through integration in stroke prediction scores such as the $\mathrm{ABC}$ and $\mathrm{CHA}_{2} \mathrm{DS}_{2}-$ VASc scores. In the present study, AF significantly predicted stroke only at ages $\geq 70$ years, with a twofold increase in stroke risk. This is in contrast to a large Danish study, showing increased stroke risks due to AF of 1.6-2.0 as early as the age of 50 years. ${ }^{32}$ Wolf $e t a l^{2}$ found no differences in stroke risk due to AF with advancing age in the Framingham Study, although the proportion of strokes attributed to AF increased from $1.5 \%$ at ages $50-59$ years to $23 \%$ at ages $80-89 .{ }^{2}$ Oral anticoagulation or alternative ways of stroke risk reduction need to be addressed thoroughly in all age groups, but with an emphasis in older patients.
We found that $\mathrm{AF}$ significantly increased the risk of death at ages $\geq 60$ years, with a 1.8-fold to 2.6-fold increase in mortality in AF patients. A large cohort study of Japanese men and women showed similar mortality rates in relation to $\mathrm{AF}$ in the age range $40-69$ years. ${ }^{33}$ However, AF itself accounts for a comparatively small fraction of deaths worldwide, ${ }^{1}$ since the mortality risk in $\mathrm{AF}$ patients is attributed to other causes such as heart failure and stroke. ${ }^{34}$

\section{Limitations and strengths}

The included cohorts originate from population-based studies to which entire birth cohorts or random samples were invited; however, we cannot rule out selection bias due to selective participation. Previous studies indicate that non-participants may have lower socioeconomic status and higher prevalences of non-communicable diseases. ${ }^{35}$ Participation among the oldest old was lower, which prompted us to merge data on participants $>70$ years, limiting knowledge among this specific group. We did not stratify by sex, as sex differences in AF risk factors were addressed previously in a similar cohort. ${ }^{17}$

Furthermore, misclassification and lack of valid data on potential residual confounders such as physical activity may lead to biased estimates. Combining data from heterogeneous cohorts may introduce misclassification, although we attempted to improve the goodnessof-fit of the Cox models by adjusting for study cohort. Data on risk factors were available only at baseline, and procedures and thresholds may have changed during follow-up. Information on AF is derived from hospital discharge records and individuals with paroxysmal AF and silent $\mathrm{AF}$ are probably underrepresented, which may weaken the associations, although validation studies show high positive and negative predictive values for AF diagnoses from diagnosis registers. ${ }^{36} 37$

Strengths of this study include the prospective design with long-term follow-up, rigorously harmonised risk factors and endpoints and a large sample size, representing Europe from North to South.

\section{CONCLUSIONS}

In this cohort of adult and elderly European men and women, incidence of AF increased significantly with age. High BMI, hypertension, high alcohol consumption and previous stroke or MI predicted risk of AF across age groups. In particular, the PAF of BMI was substantial at ages 40-69 years, and a large proportion of the AF risk was attributed to hypertension, with an age-related increase. Therefore, preventive measures should target risk factors rigorously from middle-aged to older individuals. Newly diagnosed $\mathrm{AF}$ was associated with a high risk of stroke and mortality in risk factor adjusted analyses, in particular at older age. Our study, thus, emphasises the potential role of risk factor control for prevention and adequate patient management after $\mathrm{AF}$ diagnosis in the older age groups, including the oldest old. 


\section{Author affiliations}

${ }^{1}$ School of Sport Sciences, UiT The Arctic University of Norway, Troms $\emptyset$, Norway

${ }^{2}$ Department of Cardiology, University Heart and Vascular Center Hamburg, Hamburg, Germany

${ }^{3}$ Center for Clinical Research and Prevention, Bispebjerg and Frederiksberg University Hospital, Copenhagen, Denmark

${ }^{4}$ Department of Clinical Medicine, University of Copenhagen, Copenhagen, Denmark ${ }^{5}$ Department of Public Health and Clinical Medicine, Umeå University, Umeå,

Sweden

${ }^{6}$ Department of Public Health Solutions, Finnish Institute for Health and Welfare, Helsinki, Finland

${ }^{7}$ Research Center in Epidemiology and Preventive Medicine (EPIMED), University of Insubria, Varese, Italy

${ }^{8}$ Department of Epidemiology and Prevention, IRCCS NEUROMED, Pozzilli (IS), Italy

${ }^{9}$ Department of Medicine, Turku University Hospital and University of Turku, Turku, Finland

${ }^{10}$ Department of Cardiology, Rigshospitalet, University of Copenhagen, Copenhagen, Denmark

${ }^{11}$ Department of Community Medicine, UiT The Arctic University of Norway, Troms $\emptyset$, Norway

${ }^{12}$ Department of Clinical Medicine, UiT The Arctic University of Norway, Tromsø, Norway

${ }^{13}$ Department of Neurology, University Hospital of North Norway, Troms $\emptyset$, Norway

${ }^{14}$ German Center for Cardiovascular Research (DZHK), partner site Hamburg/Kiel/ Lübeck, Hamburg, Germany

\section{Twitter Bente Morseth @MorsethBente}

Contributors RBS, BM, BG and M-LL contributed to the conception and the design of the work. M-LL, BG, BM, RBS, KK, VS, TJN, LI, SC, JKKV, IN, EBM, AL, TW, SS and $\mathrm{LJ}$ contributed to the acquisition, analysis or interpretation of data for the work. $\mathrm{BM}, \mathrm{RBS}, \mathrm{BG}$ and M-LL drafted the manuscript. All authors critically revised the manuscript. All gave final approval and agreed to be accountable for all aspects of work ensuring integrity and accuracy.

Funding The MORGAM Project has received funding from EU projects MORGAM (Biomed, BMH4-CT98-3183), GenomEUtwin (FP5, QLG2-CT-2002-01254), ENGAGE (FP7, HEALTH-F4-2007-201413), CHANCES (FP7, HEALTH-F3-2010-242244), BiomarCaRE (FP7, HEALTH-F2-2011-278913), euCanSHare (Horizon 2020, No. 825903) and AFFECT-EU (Horizon 2020, No. 847770); and Medical Research Council, London (G0601463, No. 80983: Biomarkers in the MORGAM Populations). This has supported central coordination, workshops and part of the activities of the MORGAM Data Centre, the MORGAM Laboratories and the MORGAM Participating Centres. TJN was supported by the Emil Aaltonen Foundation, Paavo Nurmi Foundation, Finnish Medical Foundation, and Academy of Finland (grant number 321351). RBS has received funding from the European Research Council (ERC) under the European Union's Horizon 2020 research and innovation programme under the grant agreement № 648131, from the European Union's Horizon 2020 research and innovation programme under the grant agreement No 847770 (AFFECT-EU) and German Center for Cardiovascular Research (DZHK e.V.) (81Z1710103); German Ministry of Research and Education (BMBF 01ZX1408A) and ERACoSysMed3 (031L0239).

Competing interests RBS has received lecture fees and advisory board fees from BMS/Pfizer outside this work.

Patient consent for publication Not required.

Ethics approval Each participating study was approved by the local ethics committee and/or institutional review board. All procedures performed in studies involving human participants were in accordance with the ethical standards of the institutional and/or national research committee and with the 1964 Helsinki Declaration and its later amendments or comparable ethical standards. Written or verbal informed consent was obtained from all individual participants.

Provenance and peer review Not commissioned; externally peer reviewed.

Data availability statement The data are not available in a public repository. Access to the data is restricted by the ethical approvals and the legislation of the European Union and the countries of each MORGAM study. Approval by the Principal Investigator of each cohort study and the MORGAM/BiomarCaRE Steering Group will be required for release of the data. The MORGAM Manual at https:// www.thl.fi/publications/morgam/manual/contents.htm gives more information on access to the data.

Open access This is an open access article distributed in accordance with the Creative Commons Attribution Non Commercial (CC BY-NC 4.0) license, which permits others to distribute, remix, adapt, build upon this work non-commercially, and license their derivative works on different terms, provided the original work is properly cited, appropriate credit is given, any changes made indicated, and the use is non-commercial. See: http://creativecommons.org/licenses/by-nc/4.0/.

\section{ORCID iDs}

Bente Morseth http://orcid.org/0000-0002-7973-0342

Kari Kuulasmaa http://orcid.org/0000-0003-2165-1411

Veikko Salomaa http://orcid.org/0000-0001-7563-5324

Licia lacoviello http://orcid.org/0000-0003-0514-5885

Simona Costanzo http://orcid.org/0000-0003-4569-1186

Stefan Söderberg http://orcid.org/0000-0001-9225-1306

Maja-Lisa Løchen http://orcid.org/0000-0002-8532-6573

Renate B Schnabel http://orcid.org/0000-0001-7170-9509

\section{REFERENCES}

1 Chugh SS, Havmoeller R, Narayanan K, et al. Worldwide epidemiology of atrial fibrillation: a global burden of disease 2010 study. Circulation 2014;129:837-47.

2 Wolf PA, Abbott RD, Kannel WB. Atrial fibrillation as an independent risk factor for stroke: the Framingham study. Stroke 1991;22:983-8.

$3 \mathrm{Kim} \mathrm{MH}$, Johnston SS, Chu B-C, et al. Estimation of total incremental health care costs in patients with atrial fibrillation in the United States. Circ Cardiovasc Qual Outcomes 2011;4:313-20.

4 Benjamin EJ, Wolf PA, D'Agostino RB, et al. Impact of atrial fibrillation on the risk of death: the Framingham heart study. Circulation 1998;98:946-52.

5 Alonso A, Krijthe BP, Aspelund T, et al. Simple risk model predicts incidence of atrial fibrillation in a racially and geographically diverse population: the CHARGE-AF consortium. J Am Heart Assoc 2013;2:e000102.

6 Everett BM, Cook NR, Conen D, et al. Novel genetic markers improve measures of atrial fibrillation risk prediction. Eur Heart $J$ 2013;34:2243-51.

7 Wingerter R, Steiger N, Burrows A, et al. Impact of lifestyle modification on atrial fibrillation. Am J Cardiol 2020;125:289-97.

8 Huxley RR, Lopez FL, Folsom AR, et al. Absolute and attributable risks of atrial fibrillation in relation to optimal and borderline risk factors: the Atherosclerosis Risk in Communities (ARIC) study. Circulation 2011;123:1501-8.

9 Stefansdottir H, Aspelund T, Gudnason V, et al. Trends in the incidence and prevalence of atrial fibrillation in lceland and future projections. Europace 2011;13:1110-7.

10 Psaty BM, Manolio TA, Kuller LH, et al. Incidence of and risk factors for atrial fibrillation in older adults. Circulation 1997;96:2455-61.

11 Heeringa J, van der Kuip DAM, Hofman A, et al. Prevalence, incidence and lifetime risk of atrial fibrillation: the Rotterdam study. Eur Heart J 2006;27:949-53.

12 Kirchhof P, Benussi S, Kotecha D, et al. 2016 ESC guidelines for the management of atrial fibrillation developed in collaboration with EACTS. Eur Heart J 2016;37:2893-962.

13 Murthy VL, Abbasi SA, Siddique J, et al. Transitions in metabolic risk and long-term cardiovascular health: coronary artery risk development in young adults (CARDIA) study. J Am Heart Assoc 2016;5. doi:10.1161/JAHA.116.003934. [Epub ahead of print: 1310 2016].

14 De With RR, Marcos EG, Van Gelder IC, et al. Atrial fibrillation progression and outcome in patients with young-onset atrial fibrillation. Europace 2018;20:1750-7.

15 Schnabel RB, Yin X, Gona P, et al. 50 year trends in atrial fibrillation prevalence, incidence, risk factors, and mortality in the Framingham heart study: a cohort study. Lancet 2015;386:154-62.

16 Magnussen C, Ojeda FM, Wild PS, et al. Atrial fibrillation manifestations risk factors and sex differences in a populationbased cohort (from the Gutenberg health study). Am J Cardiol 2018;122:76-82.

17 Magnussen C, Niiranen TJ, Ojeda FM, et al. Sex differences and similarities in atrial fibrillation epidemiology, risk factors, and mortality in community cohorts: results from the BiomarCaRE Consortium (biomarker for cardiovascular risk assessment in Europe). Circulation 2017;136:1588-97.

18 Zeller T, Hughes M, Tuovinen T, et al. BiomarCaRE: rationale and design of the European BiomarCaRE project including 300,000 
participants from 13 European countries. Eur J Epidemiol 2014;29:777-90.

19 Gerdes LU, Brønnum-Hansen H, Madsen M, et al. Trends in selected biological risk factors for cardiovascular diseases in the Danish MONICA population, 1982-1992. J Clin Epidemiol 2000;53:427-34.

20 Borodulin K, Tolonen H, Jousilahti P, et al. Cohort profile: the National FINRISK study. Int J Epidemiol 2018;47:696-696i.

21 lacoviello L, Rago L, Costanzo S, et al. The Moli-sani project: computerized ECG database in a population-based cohort study. J Electrocardiol 2012;45:684-9.

22 Lindroth M, Lundqvist R, Lilja M, et al. Cardiovascular risk factors differ between rural and urban Sweden: the 2009 northern Sweden MONICA cohort. BMC Public Health 2014;14:825

23 Jacobsen BK, Eggen AE, Mathiesen EB, et al. Cohort profile: the Tromso study. Int J Epidemiol 2012;41:961-7.

24 Evans A, Salomaa V, Kulathinal S, et al. MORGAM (an international pooling of cardiovascular cohorts). Int J Epidemiol 2005;34:21-7.

25 Magnussen C, Niiranen TJ, Ojeda FM, et al. Sex-Specific Epidemiology of Heart Failure Risk and Mortality in Europe: Results From the BiomarCaRE Consortium. JACC Heart Fail 2019;7:204-13.

26 Benichou J. A review of adjusted estimators of attributable risk. Stat Methods Med Res 2001;10:195-216.

27 Kavousi M. Differences in epidemiology and risk factors for atrial fibrillation between women and men. Front Cardiovasc Med 2020;7:3.

28 Lau DH, Nattel S, Kalman JM, et al. Modifiable risk factors and atrial fibrillation. Circulation 2017:136:583-96.

$29 \mathrm{Kim}$ YG, Han KD, Choi Jl. Non-genetic risk factors for atrial fibrillation are equally important in both young and old age: a nationwide population-based study. Eur J Prev Cardiol 2020;2047487320915664.

30 Bose A, O'Neal WT, Wu C, et al. Sex Differences in Risk Factors for Incident Atrial Fibrillation (from the Reasons for Geographic and Racial Differences in Stroke [REGARDS] Study). Am J Cardiol 2019;123:1453-7.

31 Berg DD, Ruff CT, Jarolim P, et al. Performance of the ABC scores for assessing the risk of stroke or systemic embolism and bleeding in patients with atrial fibrillation in engage AF-TIMI 48. Circulation 2019;139:760-71.

32 Frost L, Vukelic Andersen L, Godtfredsen J, et al. Age and risk of stroke in atrial fibrillation: evidence for guidelines? Neuroepidemiology 2007;28:109-15.

33 Sairenchi T, Yamagishi K, Iso H, et al. Age-Specific Impact of Atrial Fibrillation on Cardiovascular Mortality Among Japanese Men and Women (The Ibaraki Prefectural Health Study [IPHS]). Am J Cardiol 2019;124:1413-9.

34 Healey JS, Oldgren J, Ezekowitz M, et al. Occurrence of death and stroke in patients in 47 countries 1 year after presenting with atrial fibrillation: a cohort study. Lancet 2016;388:1161-9.

35 Langhammer A, Krokstad S, Romundstad P, et al. The HUNT study: participation is associated with survival and depends on socioeconomic status, diseases and symptoms. BMC Med Res Methodol 2012;12:143.

36 Norberg J, Bäckström S, Jansson J-H, et al. Estimating the prevalence of atrial fibrillation in a general population using validated electronic health data. Clin Epidemiol 2013;5:475-81.

37 Malmo V, Langhammer A, Bønaa KH, et al. Validation of self-reported and hospital-diagnosed atrial fibrillation: the HUNT study. Clin Epidemiol 2016;8:185-93. 